\title{
An optimized link state routing protocol based on a cross layer design for wireless mesh network
}

\author{
Prof. Rekha Patil. ${ }^{1}$, Abhishek $^{2}$ \\ ${ }^{1}$ Associate Professor, Department of computer science and engineering, Poojya doddappa Appa College of \\ engineering, Gulbarga, Karnataka, India, \\ ${ }^{2}$ Department of computer science and engineering, Poojya doddappa Appa College of engineering, Gulbarga,
} Karnataka, India,

\begin{abstract}
Analysis of routing in Mesh Network reveals that Proactive routes are fast but suffers vulnerability of route failure under high mobility. Reactive routes on the other hand add extra overhead in the network for obtaining a route before every communication session. As Link state routing provides a Network Map at each node, It is well suited for a mesh network. However due to uncertain demand in the mesh network, link states needs frequent updation and refreshing. A refreshing phase halts current session thus adding latency to the session. In order to avoid this pitfall we propose a unique Cross layer Based Link State Routing for Mesh network. Nodes keep monitoring the link quality as an when a packet is received in a link. Signal to Noise Ratio and Received power is measured at the MAC layer. Any change in the stored value raises an event which is read directly by the network layer. Once Network layer gets the notification, it automatically updates the route table entry with new metric values. Thus there is no specific refresh phase and nodes automatically update the links and route cache. Once a node realizes that the link quality with its next hop has degraded and a better one is available, it opts a handover of the connection called vertical handover. Thus proposed system provides seamless connectivity under varying load and mobility.
\end{abstract}

Keywords: Cross layer routing, Link state routing protocol, Wireless mesh networks (WMNs).

\section{Introduction}

Wireless mesh networks (WMNs) have emerged as a key technology for next-generation wireless networking. Because of their advantages over other wireless networks, WMNs are undergoing rapid progress and inspiring numerous applications. However, many technical issues still exist in this field. In order to provide a better understanding of the research challenges of WMNs, here author has presented a detailed investigation of current state-of-the-art protocols and algorithms for WMNs. Open research issues in all protocol layers are also discussed, with an objective to spark new research interests in this field In WMNs, nodes are comprised of mesh routers and mesh clients. Each node operates not only as a host but also as a router, forwarding packets on behalf of other nodes that may not be within direct wireless transmission range of their destinations. A WMN is dynamically self-organized and self-configured, with the nodes in the network automatically establishing and maintaining mesh connectivity among themselves (creating, in effect, an ad hoc network). This feature brings many advantages to WMNs such as low up-front cost, easy network maintenance, robustness, and reliable service coverage. Conventional nodes (e.g., desktops, laptops, PDAs, Pocket PCs, phones, etc.) equipped with wireless network interface cards (NICs) can connect directly to wireless mesh routers.

Wireless mesh networks (WMNs) are dynamically self-organized and self-configured, with the nodes in the network automatically establishing an ad hoc network and maintaining the mesh connectivity. WMNs are comprised of two types of nodes: mesh routers and mesh clients. Other than the routing capability for gateway/bridge functions as in a conventional wireless router, a mesh router contains additional routing functions to support mesh networking. Through multi-hop communications, the same coverage can be achieved by a mesh router with much lower transmission power. To further improve the flexibility of mesh networking, a mesh router is usually equipped with multiple wireless interfaces built on either the same or different wireless access technologies. In spite of all these differences, mesh and conventional wireless routers are usually built based on a similar hardware platform. Mesh routers have minimal mobility and form the mesh backbone for mesh clients. Thus, although mesh clients can also work as a router for mesh networking, the hardware platform and software for them can be much simpler than those for mesh routers. For example, communication protocols for mesh clients can be light-weight, gateway or bridge functions do not exist in mesh clients, and only a single wireless interface is needed in a mesh client, and so on. In addition to mesh networking among mesh routers and mesh clients, the gateway/bridge functionalities in mesh routers enable the integration of WMNs with various other networks. Conventional nodes equipped with wireless network interface cards (NICs) can connect directly to WMNs through wireless mesh routers. 
Customers without wireless NICs can access WMNs by connecting to wireless mesh routers through, for example, Ethernet. Thus, WMNs will greatly help users to be always-on-line anywhere, anytime. Consequently, instead of being another type of ad-hoc networking, WMNs diversify the capabilities of ad-hoc networks. This feature brings many advantages to WMNs, such as low up-front cost, easy network maintenance, robustness, reliable service coverage, etc. Therefore, in addition to being widely accepted in the traditional application sectors of ad hoc networks, WMNs are undergoing rapid commercialization in many other application scenarios such as broadband home networking, community networking, building automation, highspeed metropolitan area networks, and enterprise networking.

WMNs support ad hoc networking, and have the capability of self-forming, self-healing, and selforganization. WMNs are multi-hop wireless networks, but with a wireless infrastructure/backbone provided by mesh routers. Mesh routers have minimal mobility and perform dedicated routing and configuration, which significantly decreases the load of mesh clients and other end nodes. Mobility of end nodes is supported easily through the wireless infrastructure. Mesh routers integrate heterogeneous networks, including both wired and wireless. Thus, multiple types of network access exist in WMNs. Power-consumption constraints are different for mesh routers and mesh clients. WMNs are not stand-alone and need to be compatible and interoperable with other wireless networks.

The paper is organized as follows section I Introduction, section II related work, section III represents Methodology, system design and implementation algorithm, section IV represents Results and Discussion, section $\mathbf{V}$ finally conclusion.

\section{Related Work}

Wireless mesh network (WMN) is a novel kind of distributed broadband network architecture, of which the key idea is that every node in the network can be the access In recent years, the research approach of integrating cross-layer idea into the WMN routing protocol design has made obvious progress, which can increase the utilization of network resources to a large extent and enhance the Qos guarantee to users.

Point and the router at the same time [1]. This approach has obtained the recognition of a growing number of scholars and research institutions. The traditional minimum hop based routing protocol $[2]$ has the flaws that it cannot effectively control congestion, has poor fairness, and cannot realize load balance. Reference [3] proposed a capacity-aware routing (CAR) protocol, which adopted a routing metric called bottleneck link capacity (BLC). This metric can increase the network throughput and reduce the end-to-end delay to a certain degree by the cross-layer operation of considering the link interference, the link load, and other link quality information.

Proposed a QoS-aware routing with a congestion control and load balancing protocol (QRCCLB) [4], which, by introducing the cross-layer operation, takes dynamic source routing protocol in an Ad hoc network as the prototype and can make the network traffic bypass the network's business hotspot, thus achieve the effect of congestion control and load balance. In [5], a wireless fidelity Ad hoc on-demand distance vector (WiFiAODV) routing protocol was proposed, which fully exploits the adaptive rate switching mechanism of IEEE802.11 by introducing the cross-layer mechanism, in which nodes use the data transmission rate of the physical layer as a metric and are able to establish a route with a high data rate and low transmission delay.

proposed an integrated metrics based extended dynamic source routing method (EDSR) [6], which uses the cross-layer design to provide the frame delivery rate, extra bandwidth, and the node load of the media access control (MAC) layer for the network layer routing algorithm, thus improving the throughput rate and load balance capability of the network and satisfying users' QoS requirements by promoting the network's overall performance. These routing protocols are all proposed based on existing on-demand routing protocols of Ad hoc network, such as dynamic source routing, and Ad hoc on-demand distance vector routing. But for WMN, the network node is relatively fixed. Only node failures, as well as joining, leaving, and the uncertainty of wireless links will result in changes in the network topology. The change rate of network topology is far below the arrival rate of the data flow and the main business in WMN is the Internet business with certain delay requirements. These routing protocols have jumped out of the traditional route of taking the minimum hop as the routing metric and introduced the idea of cross-layer design. But there is a lack of systemic knowledge for the cross-layer design of WMN, in which the implementation process is complex and the practicality is low

The design of a CL-OLSR protocol for WMN based on optimized link state routing (OLSR) protocol. CL-OLSR based on [6] refines the idea of cross-layer design, and proposes a brand-new routing metric CLM applicable to WMN. This routing metric [7] takes into account four cross-layer factors: node available bandwidth, node balance, link delivery rate, and link interference by introducing the cross-layer operation mechanism. Through considering these four factors, the route selection is optimized to a large extent, the network throughput is improved and the goal of load balance is achieved. Compared with the on-demand routing, CL-OLSR is a proactive routing protocol[8] of hop-by-hop forwarding, which is more suitable for 
approximately static topological properties of WMN, and satisfies the requirements of low delay of the traffic flow. Moreover, the algorithm is relatively simple and practical.

\section{Methodology}

The overview of the project methodology is explained in brief as follows.

- Every Node Measure the SINR and Received signal strength for every received packet. This value is updated in Neighbor Table by Network layer if there is a significant variation with past value.

- A route is established using OLSR which takes into account of current state of links

- In the Middle of a session if a node tracks that better access point/relay node is available with better link quality, then requests for a change in connection.

- Transmission starts through this path upon link switch over.

- Thus routing is through the best link. Best link is always maintained without rerouting. MAC layer parameter like Packet Rate. In physical layer calculate Power Loss.

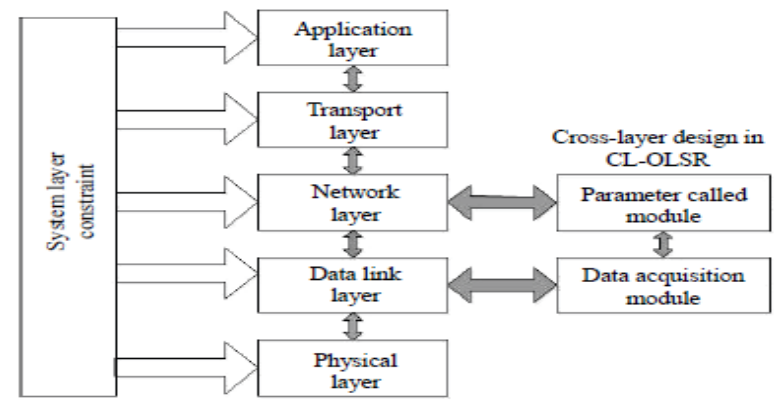

Fig. 1 Cross-layer module

Proposed Work

Routing is simply the function of knowing which path to take in order to deliver the data from one end to another. It must include an addressing scheme and a routing protocol. To put it most simply, if the addressing scheme consisted of house addresses, then a knowledgeable postman would be the routing protocol. Routing protocols are worked hard in a mesh, particularly if it is mobile. We can imagine our postman's job if houses were regularly to disappear and re-appear somewhere else, or if they are all moved constantly in different directions. This is the nature of the mobile routing protocol challenge. Routing protocols must be efficient and must either keep their knowledge proactively up-to-date, or be quickly reactive when routes are required. Which of these two routing approaches is better depends on the behaviour of the mesh users. And the traffic which must be carried. Each mesh node must participate in the routing and relay of other's traffic. Every node is a router In an Mesh network environment, each node participates within the network not only as a possible source and sink of traffic but, also as a relay and a router, enabling the forwarding of traffic between nodes in the network. Thus, in a mesh, each and every node needs to know what the route traffic needs to follow in order to reach its destination. This is directly analogous to router behaviour in the internet, although the big difference is that not all internet nodes need to be routers. It follows mobility adds volatility to the routing problem.

Every node is a relay It is always necessary for nodes to act as relays to form a mesh. This is essential but has several consequences.

Firstly, by acting as a relay, anode takes on a workload over and above that needed to support its user's requirements. This accepted consensus from both academic and commercial published work is that nodes often need to be able to relay traffic of a volume a few times above that of their own service, i.e. they must handle not only their user generated traffic but potentially that of several other users as well. This implies that meshes will require more capable user nodes than otherwise equivalent cellular systems. Secondly, a consequence of having a service level which depends on the performance of user nodes is an increased difficulty in maintenance and upgrade. For example with the introduction of EDGE onto GSM it was possible to upgrade base stations, and then allow subscribers to sign up to the new services as and when required, if at all. With a mesh system, users are dependent on the installed base of fellow subscribers and so new services cannot be provided unless all or at least a substantial fraction of existing users are persuaded to upgrade their units. Proactive and reactive routing Mesh routing protocols are used in environments where there is not necessarily a well-controlled infrastructure network but where there is a common routing policy. There are two main categories of routing: proactive and reactive. Proactive routing protocols are based on the 'normal' routing protocols used in wired networks, such as today's internet. Algorithms based on distance vector and link state are common. Distance vector often uses the number of hops as a metric to minimise when selecting the best route, but can also go beyond this to consider more parameters, for example link bandwidth and delay. This is used to construct a route table, which is shared with other routers. 
Link state operation is more complex and requires each router to build its own map of the network. Thus, in proactive protocols, there is an attempt to build locally, at each node, a picture of roots within the network before they are required for use. The routing tables are usually built periodically through the normal operation of the protocol exchanging routing update packets. In normal operation, this has advantage that the routes are already pre-computed and so packet forwarding can take place as soon as a packet for a particular destination appears at a node. The drawback is that routes may be calculated and re-calculated (for e.g. due to node mobility) when they are actually not required for data. This wastes bandwidth and, for mobile nodes, also waste battery power via the sending and receiving of unnecessary routing updates. Reactive routing takes an alternative approach by building routes only upon demand. It may also cache route information according to some short time-out or stale-ness policy. Cached routes can be used as required, but if a route is not known then it has to be 'discovered'. This has advantage that routes are only evaluated when needed, although this approach adds latency to packet forwarding when routes are not already known. Generally, the reactive routing approach is the one that has received most attention in the adhoc networking community.

\section{Sequence Numbers}

A difficulty with flooding, as described so far, is that when all routers have received all LSAs, the flooding must stop. A time-to-live value in the packets could simply be relied on to expire, but it is hardly efficient to permit LSAs to wander the network until they expire. Take the network in Figure 2. Subnet 172.22.4.0 at Router A has failed, and A has flooded an LSA to its neighbors B and D, advertising the new state of the link. B and D dutifully flood to their neighbors, and so on.

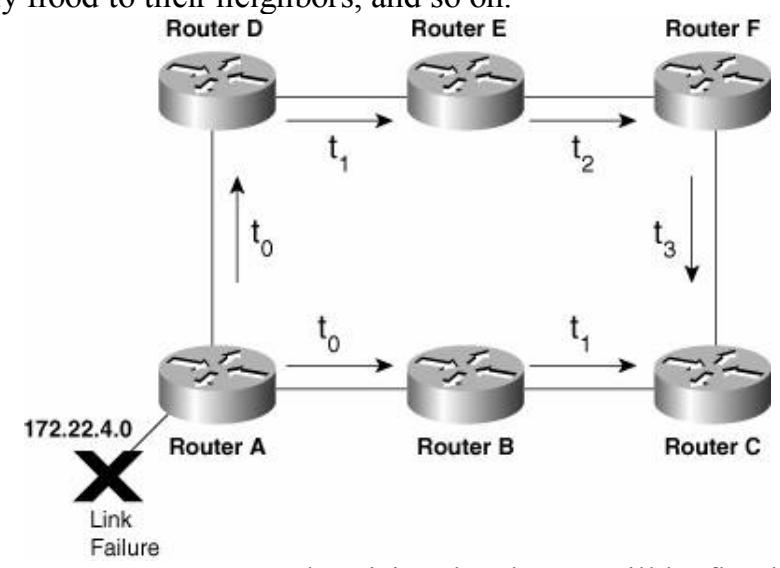

Fig. 2 When a topology change occurs, LSAs advertising the change will be flooded throughout the network.

Look next at what happens at Router C. An LSA arrives from Router B at time t1, is entered into C's topological database, and is forwarded to Router F. At some later time t3, another copy of the same LSA arrives from the longer A-D-E-F-C route. Router C sees that it already has the LSA in its database; the question is, should C forward this LSA to Router B? The answer is no because B has already received the advertisement. Router $\mathrm{C}$ knows this because the sequence number of the LSA it received from Router $\mathrm{F}$ is the same as the sequence number of the LSA it received earlier from Router B. When Router A sent out the LSA, it included an identical sequence number in each copy. This sequence number is recorded in the routers' topological databases along with the rest of the LSA; when a router receives an LSA that is already in the database and its sequence number is the same, the received information is discarded. If the information is the same but the sequence number is greater, the received information and new sequence number are entered into the database and the LSA is flooded. In this way, flooding is abated when all routers have seen a copy of the most recent LSA.

As described so far, it seems that routers could merely verify that their link state databases contain the same LSA as the newly received LSA and make a flood/discard decision based on that information, without needing a sequence number. But imagine that immediately after Figure 2's network 172.22.4.0 failed, it came back up. Router A might send out an LSA advertising the network as down, with a sequence number of 166; then it sends out a new LSA announcing the same network as up, with a sequence number of 167 . Router C receives the down LSA and then the up LSA from the A-B-C path, but then it receives a delayed down LSA from the A-D-E-F-C path. Without the sequence numbers, $\mathrm{C}$ would not know whether or not to believe the delayed down LSA. With sequence numbers, C's database will indicate that the information from Router A has a sequence number of 167 ; the late LSA has a sequence number of 166 and is therefore recognized as old information and discarded. 
Algorithm

Step 1: The number of nodes are initialized over an area of $a=X * Y$ mts.

Step 2: Any node is represented by layer $\mathrm{L}=\{\mathrm{A}, \mathrm{N}, \mathrm{P}, \mathrm{LL}, \mathrm{NB}\}$

Where,

$\mathrm{A}=$ application layer

$\mathrm{N}=$ network layer

$\mathrm{P}=$ physical layer

$\mathrm{LL}=$ link layer

$\mathrm{NB}=$ notification board.

Step 3: At the time of initialization each node Ni broadcast its equal li to its neighbours. Upon receiving li by $\mathrm{Nj}$. $\mathrm{Nj}$ calculates link quality Qij makes an table entry P.IJ $=\{\mathrm{i} . \mathrm{j}$, li Qij $\}$

Step 4: At the saturation state any routes RI finds a path to all the nodes either directly or through any other arbitrary router $\mathrm{Rj}$ by iterating in $\mathrm{T}$.

Step 5: Every router RI exchanges every possible route with every other route RJ.

Step 6: Association phase - where NI closure to RI request for an association which is replied by RI.

Step 7: Request phase - NI generates data packets for destination d.

Step 8: Forwarding phase - upon receiving packet from NI to d

.RI finds route from $\mathrm{T}$ and forwards to the next hop.

Step 9: Topology change phase - any node NI moves to a next location Xi2, Yi2 from its current location Xi1 ,Yil at a speed Vi using linear mobility model.

Step 10: Link quality change -The above event results in change of link quality measured at $11 \mathrm{j}$ of a node $\mathrm{J}$ initiating RE routing request.

Step 11: Node updates $\mathrm{T}$ and goes to saturation phase for obtaining new route. Further packets are forwarded through new path

\section{Results And Discussion}

wmn is composed of mesh routers and clients. The mobility of the node have significant impanct on the performance of the system.global route cache keeps a record of the network map based on the signal to noise ratio and the received power. Later routing is done to achive max throughput and to reduce the delay.

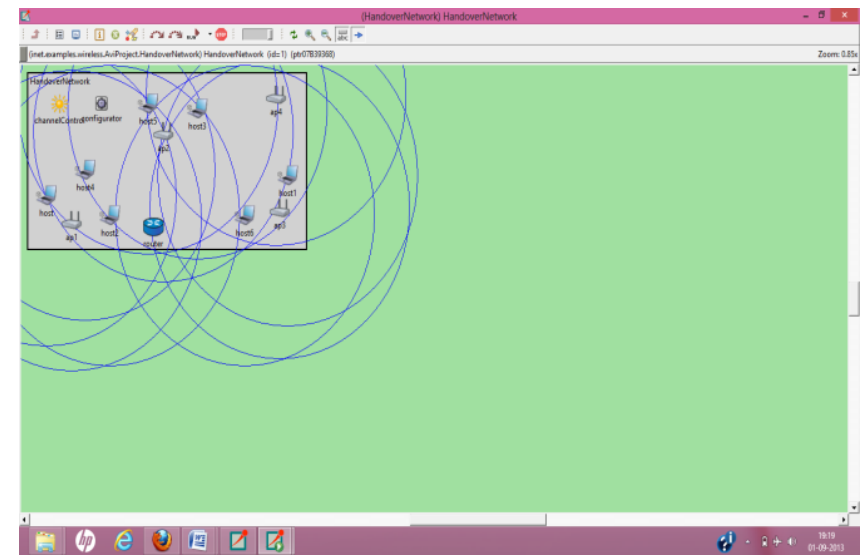

Fig. 3 Hand over networks

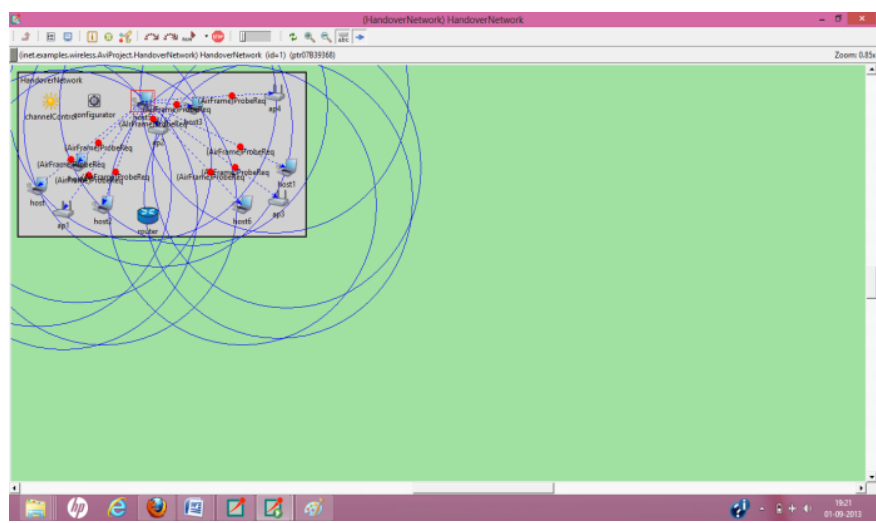

Fig. 4 Hand over networks with airframe packets 


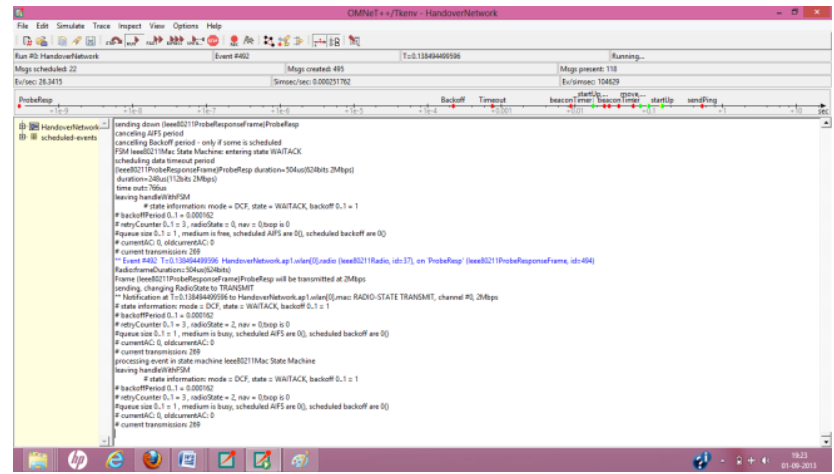

Fig. 5 Performance measurement at each node upon receiving packet

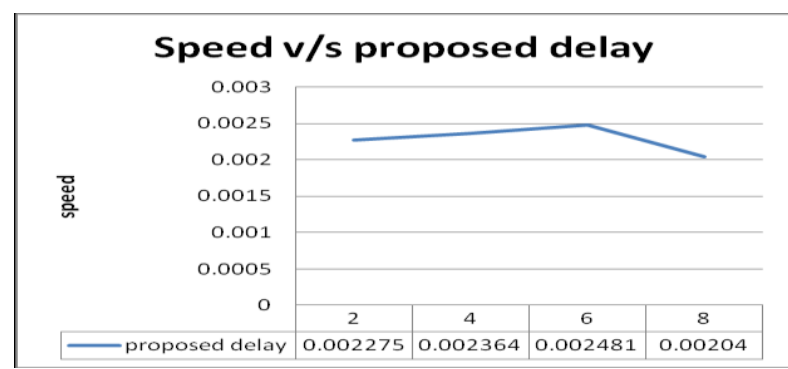

Here delay refers to the time taken for a packet to be transmitted across a network from source to destination Speed Vs proposed delay is plotted in the above fig. The different delays are queuing delay, propagation delay, processing delay, processing time. The delay is calculated for different number of nodes. As shown in figure the end to end delays compared with existing ETX method, which shows that the delay is less for the proposed bandwidth system as the speed is increased in regular interval

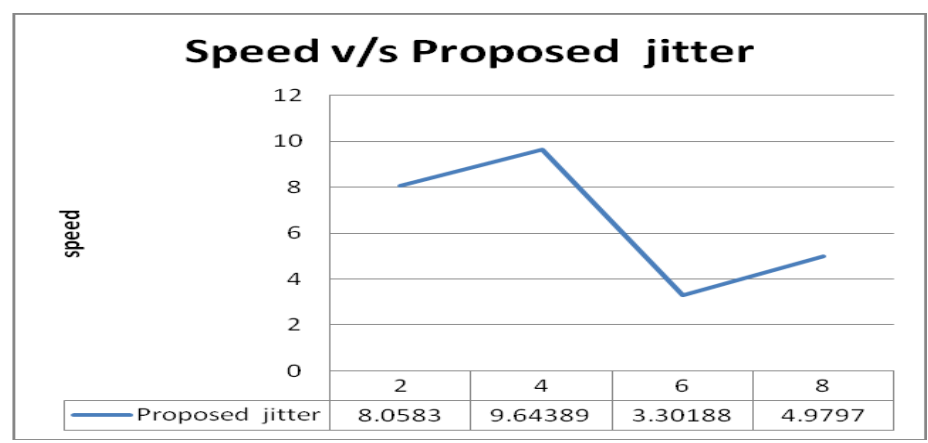

Jitter is the variation in time between packets arriving, caused by network congestion, time drift, or route changes. A jitter buffer can be used to handle jitter. The types of jitter are random jitter and deterministic jitter. Jitter is defined as a variation in the delay of received packets. At the sending side, packet are sent in a continuous stream with the packets spaced evenly apart. Due to network congestion, improper queuing, or configuration errors, this steady stream can become lumpy, or the delay between each packet can vary instead of remaining constant. The data packets forwarding overhead increases as numbers of node increases. The jitter occurs because of traffic, interference etc. also while sending more data in the routing path the data packet overhead is occurred. Since jitter is less for the proposed system.

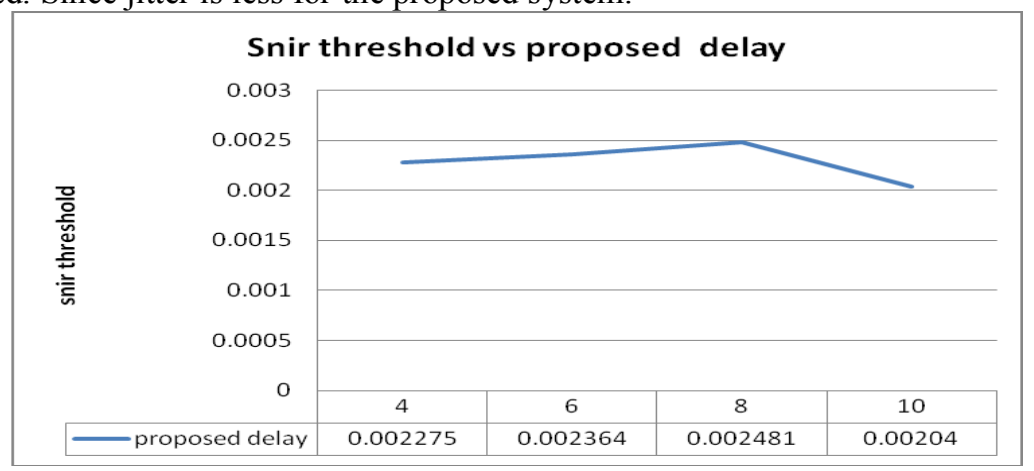

Threshold is the minimum, of the maximum value to be achieved, here the sinr gradually decreases. 


\section{Conclusion}

In this paper we show that link quality in WMN varies Due to factors like mobility, energy consumption, and power losses. Variation in link quality results in fluctuations in packet delivery ration, latency and other performances. So under link variations, routes cannot be considered as stable. Therefore incorporating link quality and deriving a suitable technique to include the same as cost metric in routing is essential. There are several techniques which estimates the link quality based on either movement or power loss. As SINR and received power directly or indirectly affects all the other parameters, we have considered SINR based link quality metric for Link State routing and vertical handoff in WMN

Here in this work we measure link stability as consistency of data and control packet rate in the links. Links with consistent rates irrespective of high or low are considered as more stable Links. Through OLSR we find the routes that incorporate the most stable links. Further if the current router's link fails or degrades, the next best router is selected. Result shows that the technique results in better routing, energy, Control Overhead under different link variability constraints like high mobility.

\section{References}

[1] J. Ren, "Wireless mesh network resource allocation and congestion control algorithm research," Ph.D. dissertation, Beijing Jiaotong University, Beijing, 2010 (in Chinese)

[2] S. Waharte, R. Boutaba, Y. Iraqi, and B. Ishibashi, "Routing protocols in wireless mesh networks: challenges and design considerations," Multimedia Tools and Applications, vol. 29, no. 3, pp. 285-303, 2006.

[3] T.-H. Liu and W.-J. Liao, "Capacity-aware routing in multi-channel multi-rate wireless mesh networks," in Proc. of 2006 IEEE International Conf. on Communications, Istanbul, 2006, pp. 1971-1976.

[4] W. Song and X.-M. Fang, "Routing with congestion control and load balancing in wireless mesh networks," in Proc. of the 6th International Conf. on ITS Telecommunications, Chengdu, 2006, pp. 719-724.

[5] Y.-F. Wai, Y. Zhang, M. Song, and J. Song, "An improved AODV routing protocol for WiFi mesh networks," Journal of Beijing University of Posts and Telecommunication, vol. 30, no. 4, pp. 120-124, 2007 (in Chinese).

[6] Q. Shen and X.-M. Fang, "An integrated metrics based extended dynamic source routing protocol in wireless mesh networks," in Proc. of International Conf. on Communications, Circuits and Systems, Guilin, 2007, pp. 1457-1461.

[7] Y.-L. Yang, J. Wang, and R. Kravets, "Designing routing metrics for mesh networks," in Proc. of the IEEE Workshop on Wireless Mesh Networks (WiMesh), Santa Clara, 2005, pp. 1-9.

[8] P. Jacquet, P. Muhlethaler, T. Clausen, A. Laouiti, A. Qayyum, and L. Viennot, "Optimized link state routing protocol for ad hoc networks," IEEE International Multi Topoic Conf., Lahore, 2001, pp. 62-68 